\title{
Fever Exacerbating Ventricular Fibrillation in Early Repolarization Syndrome
}

\author{
J. Mauricio Sanchez ${ }^{1^{*}}$, Thomas Kurian ${ }^{2}$, Amit Doshi ${ }^{2}$ and Stephen Pieper ${ }^{1}$ \\ ${ }^{1}$ Section of Electrophysiology, Department of Cardiovascular Medicine, The Heart Health Center - St. Louis, Missouri, USA \\ ${ }^{2}$ Section of Electrophysiology, Department of Cardiovascular Medicine, Mercy Clinic - St. Louis, Missouri, USA
}

"Corresponding author: J.Mauricio Sanchez, Section of Electrophysiology, Department of Cardiovascular Medicine, The Heart Health Center - St. Louis, Missouri, USA, Tel: 314-991-6969; E-mail: jsanchez@hearthealthcenter.com

Rec date: March 11, 2015; Acc date: April 29, 2016; Pub date: May 10, 2016

Copyright: ( 2016 Sanchez JM, et al. This is an open-access article distributed under the terms of the Creative Commons Attribution License, which permits unrestricted use, distribution, and reproduction in any medium, provided the original author and source are credited.

\begin{abstract}
Early repolarization (ER) syndrome is characterized by $\geq 1 \mathrm{mV}$ J-point elevation in 2 contiguous inferior and/or lateral ECG leads in a patient resuscitated from otherwise unexplained VF or polymorphic VT. J-point elevation in these patients may have slurring or notching of the end of the QRS complex. While alterations in temperature have been known to effect other arrhythmia disorders, it has not been described with ER syndrome. We report a patient with ER syndrome which manifest VF after febrile response to severe burns resulting from occupational accident.
\end{abstract}

Keywords: Early repolarization syndrome; Fever; Sudden cardiac death; Ventricular fibrillation

\section{Introduction}

Early repolarization (ER) syndrome is characterized by $\geq 1 \mathrm{mV} \mathrm{J}-$ point elevation in 2 contiguous inferior and/or lateral ECG leads in a patient resuscitated from otherwise unexplained VF or polymorphic VT [1]. J-point elevation in these patients may have slurring or notching of the end of the QRS complex. While the ER ECG pattern was previously thought benign [2], recent studies have demonstrated an association between ER and risk for ventricular arrhythmias compared to control populations $[3,4]$. While the pathophysiology is not completely understood, it is hypothesized that the J-point elevation is a result of more pronounced phase 1 notch of the epicardial action potential compared to the endocardium resulting in a greater ventricular transmural gradient. A higher transient outward current (Ito) epicardial current relative to endocarium is thought responsible [5]. This dispersion of repolarization increases the susceptibility to phase 2 re-entry. ER has also been known to share features with Brugada syndrome and care commonly referred to as "J wave syndromes." While fever has been reported to unmask Brugada and trigger ventricular arrhythmias [6], this has not been previously reported in ER syndrome. We report a case of fever triggering VF in a patient with ER syndrome.

\section{Case Report}

A 36-year old African American male without previous medical history was admitted to the authors burn unit after sustaining burn related injuries while weather proofing his basement. He was wearing protective goggles and sustained burns to his lower extremities, perineum and upper extremities. The patient was intubated for airway protection. He underwent multiple escharotomies and skin grafting procedures. ECG on admission demonstrated normal sinus rhythm with voltage criteria for left ventricular hypertrophy. J point elevation of $1 \mathrm{mV}$ was noted on leads V2-V3 only (Figure 1). The patient was monitored on telemetry during his course within the burn unit. On hospital day 9, the patient had a VF arrest and was resuscitated with defibrillation. Telemetry demonstrated sinus tachycardia with a PVC occurring on the $\mathrm{T}$ wave in the absence of bradycardia or long-short phenomena (Figure 2). The ECG after arrest and resuscitation demonstrated anterior lateral J point elevation (Figure 3A.) with a slurring at the end of the QRS complex on the inferior leads (Figure 3B). QT intervals were within normal limits. Electrolytes were within normal limits at the time of the event. Of note, the patient was febrile with a temperature of $38.7^{\circ} \mathrm{C}$ within the hour prior to the event. The patient had family history of sudden death with his father at 51 years of age. The patient had no personal history of syncope, presyncope, or palpitations. A two-dimensional echo-Doppler cardiac evaluation demonstrated mild left ventricular hypertrophy with no ventricular obstruction and normal global and segmental ventricular function. No signs of pulmonary hypertension were noted. Coronary angiography was performed with no evidence of obstructive coronary artery disease and normal left ventricular function. The patient was diagnosed with presumptive ER syndrome. Isoproterenol was initiated intravenously for acute therapy. Review of telemetry the 24 hours preceding the VF arrest demonstrated nonsustained polymorphic VT within an hour of a febrile episode of $39.4^{\circ} \mathrm{C}$ almost exactly 24 hours prior to his arrest. Because of this, aggressive temperature control was instituted with antipyretics. The patient was then transitioned to IV quinidine until able to take oral medication. While ER pattern remained present during this burn patient's critical illness, treatment resulted in attenuation of this finding. ICD was implanted once his wounds and infectious risk allowed to be performed safely. On follow up, the patient's J point elevation had resolved. Genetic testing for patient and family has been strongly recommended but declined to date. The patient has suffered no further arrhythmic events.

\section{Discussion}

This is the first reported case of febrile induction of ventricular arrhythmia in ER syndrome. While the patient's initial presentation demonstrated more benign changes, his ECG changes later were more consistent with more malignant findings such as slurring or notching of the J wave in the inferior and lateral leads intermittently throughout his prolonged hospital course. Koncz et al., [7] demonstrated in canine wedge models that inferior wall has a higher intrinsic Ito current which 
may increase the possibility of dispersion of repolarization and thus increased susceptibility to phase 2 re-entry [7]. The patient's family history of sudden death is consistent with reports from Haissaguerre et al., [3] that describe up to $14 \%$ of ER syndrome patients with VF with a family history of sudden death.

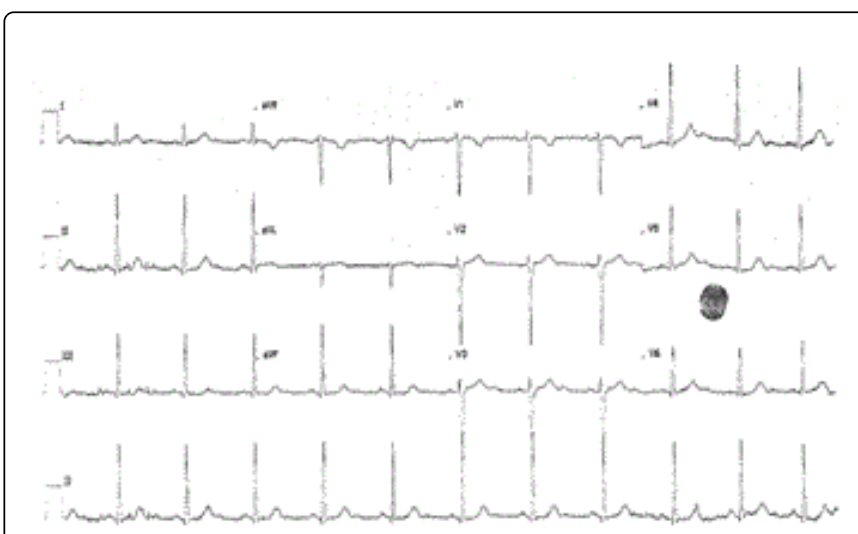

Figure 1: ECG on admission demonstrated normal sinus rhythm with voltage criteria for left ventricular hypertrophy. J point elevation of $1 \mathrm{mV}$ was noted on leads $\mathrm{V} 2-\mathrm{V} 3$ only.

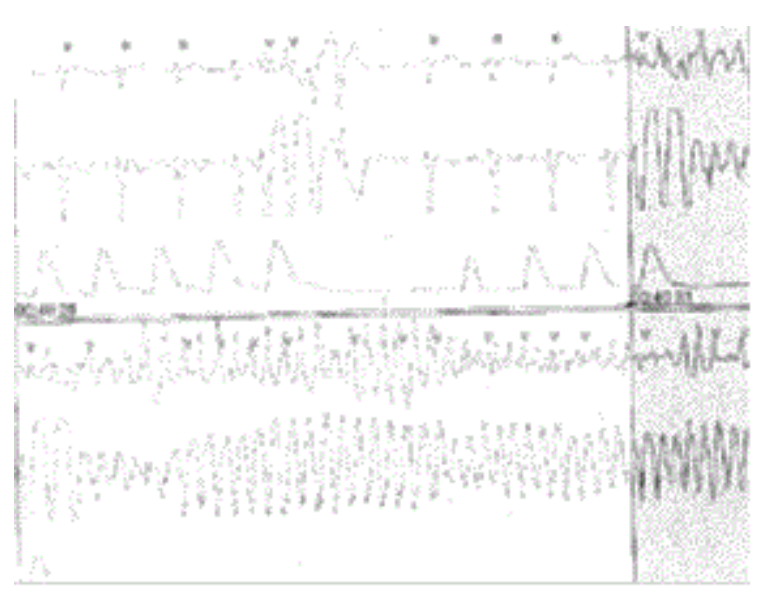

Figure 2: The patient was monitored on telemetry during his course within the burn unit. On hospital day 9, the patient had a VF arrest and was resuscitated with defibrillation. Telemetry demonstrated sinus tachycardia with a PVC occurring on the $\mathrm{T}$ wave in the absence of bradycardia or long-short phenomena.

Burn injury frequently results in elevated temperature due to metabolic and physiologic alterations as well as with infection. Temperature has been known to modulate J waves and ST-segment elevation, classically with hypothermia inducing prominent $J$ waves known as Osborn waves [8]. Fever has been reported to unmask Brugada or produce a Brugada-like ECG [6]. Temperature dependent nature of defects with the SCN5A gene has been described in association with Brugada syndrome [9]. Temperature dependent changes in current have also been hypothesized with other channels with regard to Brugada syndrome, such as the Ito channel [10]. Fever has also been described as inducing QT prolongation and ventricular events in Long QT syndrome [11]. This patient did not have a Bruagada phenotype at any point pre or post arrest. Pharmacological test was not performed.

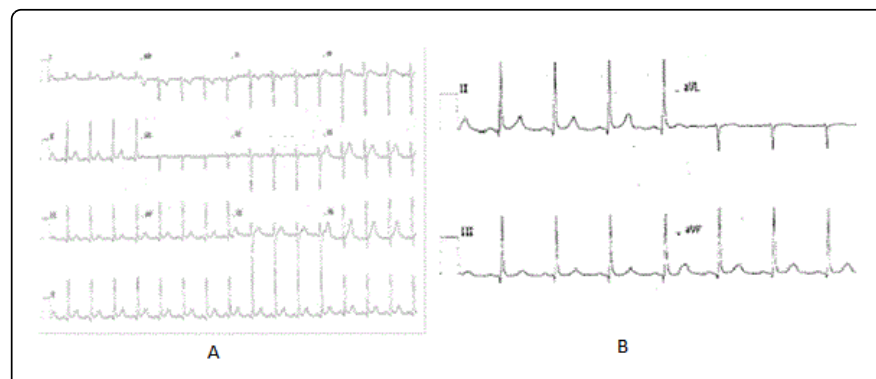

Figure 3: A and B) The ECG after arrest and resuscitation demonstrated anterior lateral J point elevation with a slurring at the end of the QRS complex on the inferior leads.

Based on our reported observation, we hypothesize that this patient's febrile temperature increased the likelihood of ventricular arrhythmia in this patient by augmenting the transmural voltage gradient by an effect on one or more ion channels. While acute therapy with isoproterenol has been reported to be effective, long-term quinidine may also be required to avoid future ICD shocks and storms of arrhythmias. A therapeutic intervention with antipyretics may be valuable in these patients.

\section{References}

1. Prior SG, Wilde AA, Horie M, Cho Y, Behr ER, et al. (2013) HRS/EHRA/ APHRS expert consensus statement on the diagnosis and management of patients with inherited primary arrhythmia syndromes. Europace 15: 1389-1406.

2. Klatsky AL, Oehm R, Cooper RA, Udaltsova N, Armstrong MA (2003) The early repolarization normal variant electrocardiogram: correlates and consequences. Am J Med 115: 171-177.

3. Haïssaguerre M, Derval N, Sacher F, Jesel L, Deisenhofer I, et al. (2008) Sudden cardiac arrest associated with early repolarization. N Engl J Med 358: 2016-2023.

4. Tikkanen JT, Anttonen O, Junttila MJ, Aro AL, Kerola T, et al. (2009) Long-term outcome associated with early repolarization on electrocardiography. N Engl J Med 361: 2529-2537.

5. Yan GX, Antzelevitch C (1996) Cellular basis for the electrocardiographic J wave. Circulation 93: 372-379.

6. Antzelevitch C, Brugada R (2002) Fever and Brugada syndrome. Pacing Clin Electrophysiol 25: 1537-1539.

7. Koncz I, Gurabi Z, Patocskai B, Panama BK, Szél T, et al. (2014) Mechanisms underlying the development of the electrocardiographic and arrhythmic manifestations of early repolarization syndrome. J Mol Cell Cardiol 68: 20-28.

8. Abbott JA, Cheitlin MD (1976) The nonspecific camel-hump sign. JAMA 235: 413-414.

9. Keller DI, Rougier JS, Kucera JP, Benammar N, Fressart V, et al. (2005) Brugada syndrome and fever: genetic and molecular characterization of patients carrying SCN5A mutations. Cardiovasc Res 67: 510-519.

10. Dumaine R, Towbin JA, Brugada P, Vatta M, Nesterenko DV, et al. (1999) Ionic mechanisms responsible for the electrocardiographic phenotype of the Brugada syndrome are temperature dependent. Circ Res 85: 803-809. 
Citation: Sanchez JM, Kurian T, Doshi A, Pieper S (2016) Fever Exacerbating Ventricular Fibrillation in Early Repolarization Syndrome. J Cardiovasc Dis Diagn S1: 006. doi:10.4172/2329-9517.1000S1-006

Page 3 of 3

11. Amin AS, Herfst LJ, Delisle BP, Klemens CA, Rook MB, et al. (2008) Fever-induced QTc prolongation and ventricular arrhythmias in individuals with type 2 congenital long QT syndrome. J Clin Invest 118: 2552-2561.

This article was originally published in a special issue, entitled: "Coronary

Artery Disease", Edited by Leonardo Silva Borges Roever 